\title{
Peculiarities of Emission Characteristics of Semiconductor Optical Amplifier with Multiple Quantum Wells
}

\author{
Di-Ku Yu, Gagik Sh. Shmavonyan, Yi-Shin Su, and *Ching-Fuh Lin \\ Graduate Institute of Electro-Optical Engineering \\ National Taiwan University \\ Taipei 106, Taiwan ROC
}

\begin{abstract}
Novel bi-directional propagation is observed in a shallow-etched bending ridge waveguide. The lasing light propagates in two different paths, straight way and bending way. The far-field pattern is quite different before and after the lasing condition is reached. Emission spectra of light emitted from two facets are also different. This is because the bidirectional guided effect of lasing mode occurs.
\end{abstract}

Keywords: bent waveguide, asymmetric multiple quantum wells, bi-directional, guiding effect, ridge waveguide

\section{Introduction}

Optical amplifier is an important device in the modern optical communication. It compensates the loss of the light in the optical fiber and makes the signal strong enough to propagate longer distance. With the advance of technology, semiconductor light emitting devices are now very efficient, stable and cheap. The most widely used active layer structure is composed of double heterojunction optical confinement layer and quantum wells layers as the light amplification layer. With the advantages of low power consumption, small chip volume, and increasingly wide bandwidth properties, semiconductor optical amplifier (SOA) is a good choice for amplification device. Semiconductor optical amplifier is slightly different from the semiconductor laser diode for its lack of resonance mechanism. There are many ways to eliminate the resonance cavity in the laser diodes. One of them is using the skills of anti-reflection coating on both facets of the laser diodes. ${ }^{1}$ Another is to fabricate the different shapes of ridge waveguides on the top of the device. However, it is difficult to design and fabricate broadband anti-reflection coating because it requires stable facility, long-term test and very stringent coating conditions. To reduce production cost and make devices have uniform performance, one will favor the bent structure ${ }^{2,3}$ or tilted structure waveguide ${ }^{4}$ for the purpose of preventing feedback.

The SOA with the bent structure waveguide will not lase by itself because light propagates to the bent side of the device will reflect to the other angle by the facet instead of normal reflection. However, we see the lasing phenomenon in the experiment on the bent structure SOA. There are two lights emitted from one facet. Bi-directional switching based on this semiconductor laser with shallow-etched bending ridge waveguide is also observed. ${ }^{5,6}$ It can be a new type of optical switching device and has two spatially distinct mode switching. The experiment shows that the current, feedback lasing wavelength, and the ambient temperature will cause multiple switching effects in this laser diode. ${ }^{6}$ In this work, we further see the light far field distribution is quite different between the device in the lasing condition and in the spontaneous-emission condition. Lasing guided mode leads to two obvious light maximum in the far field pattern, while the light distribution is more plat if the threshold current is not reached. If the feedback mechanism is used in different directions of light, the far field pattern will be changed and imply the light resonant paths. Grating is also used to feedback the desired wavelength. From the spectra, we can understand the feedback behavior of the device.

This paper is divided into several sections. The first part is the introduction, where the motivation and some basic concepts are described. The second part is about the experiments, including the device structure, the setup of our experiment and how we measure different experimental results. The third part is the results of this experiment. From the observed results, we have some discussions in the fourth section. Finally, we give a conclusion to explain the phenomenon of this novel bi-directional lasing device.

\footnotetext{
* cflin@cc.ee.ntu.edu.tw; also with Institute of Electronics Engineering and Department of Electrical Engineering.
} 


\section{Experiment}

\subsection{SOA structure}

Laser diodes are fabricated on the substrate with nonidentical quantum wells for the experiments. The QW structure has two $\mathrm{In}_{0.53} \mathrm{Ga}_{0.47} \mathrm{As}$ QWs near the p-cladding layer and three $\mathrm{In}_{0.67} \mathrm{Ga}_{0.33} \mathrm{As}_{0.72} \mathrm{P}_{0.28}$ QWs near the n-cladding layer. The QWs are separated by $\operatorname{In}_{0.86} \mathrm{Ga}_{0.14} \mathrm{As}_{0.3} \mathrm{P}_{0.7}$ barriers. At room temperature, the $\operatorname{In}_{0.53} \mathrm{Ga}_{0.47} \mathrm{As}$ QWs (QW A) and $\mathrm{In}_{0.67} \mathrm{Ga}_{0.33} \mathrm{As}_{0.72} \mathrm{P}_{0.28} \mathrm{QWs}$ (QW B) have their first quantized transition energies of $0.8 \mathrm{eV}$ and $0.954 \mathrm{eV}$, respectively. The composition and the structure of the quantum wells are shown in Fig 1.

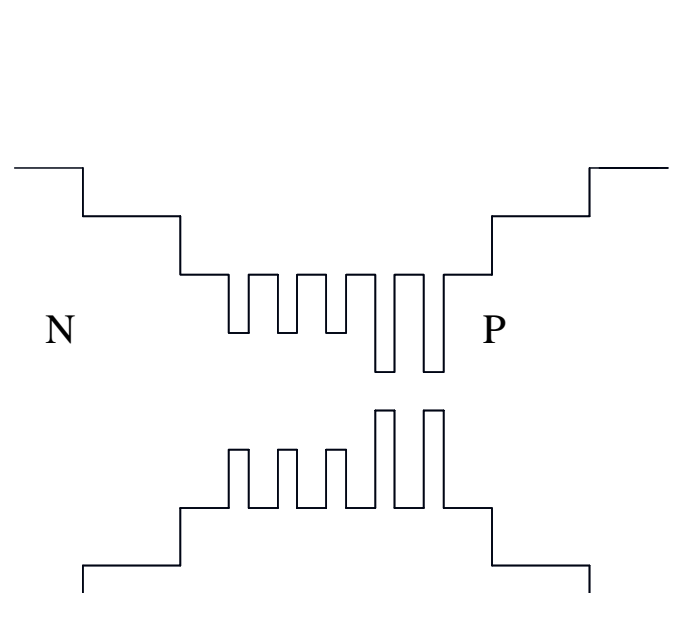

Fig 1. This is the structure and the compositions of the active layer in this device.

\begin{tabular}{|c|c|}
\hline P-InP & \\
\hline $\begin{array}{l}\text { InGaAsP quaternary @ } \\
1100 \text { nm }\end{array}$ & $75 \mathrm{~nm}$ \\
\hline $\mathrm{In}_{0.86} \mathrm{Ga}_{0.14} \mathrm{As}_{0.3} \mathrm{P}_{0.7}$ & $45 \mathrm{~nm}$ \\
\hline $\mathrm{In}_{0.53} \mathrm{Ga}_{0.47} \mathrm{As}$ & $8.7 \mathrm{~nm}$ \\
\hline $\mathrm{In}_{0.86} \mathrm{Ga}_{0.14} \mathrm{As}_{0.3} \mathrm{P}_{0.7}$ & $15 \mathrm{~nm}$ \\
\hline $\mathrm{In}_{0.53} \mathrm{Ga}_{0.47} \mathrm{As}$ & $8.7 \mathrm{~nm}$ \\
\hline $\mathrm{In}_{0.86} \mathrm{Ga}_{0.14} \mathrm{As}_{0.3} \mathrm{P}_{0.7}$ & $15 \mathrm{~nm}$ \\
\hline $\mathrm{In}_{0.67} \mathrm{Ga}_{0.33} \mathrm{As}_{0.72} \mathrm{P}_{0.28}$ & $6 \mathrm{~nm}$ \\
\hline $\mathrm{In}_{0.86} \mathrm{Ga}_{0.14} \mathrm{As}_{0.3} \mathrm{P}_{0.7}$ & $15 \mathrm{~nm}$ \\
\hline $\mathrm{In}_{0.67} \mathrm{Ga}_{0.33} \mathrm{As}_{0.72} \mathrm{P}_{0.28}$ & $6 \mathrm{~nm}$ \\
\hline $\mathrm{In}_{0.86} \mathrm{Ga}_{0.14} \mathrm{As}_{0.3} \mathrm{P}_{0.7}$ & $15 \mathrm{~nm}$ \\
\hline $\mathrm{In}_{0.67} \mathrm{Ga}_{0.33} \mathrm{As}_{0.72} \mathrm{P}_{0.28}$ & $6 \mathrm{~nm}$ \\
\hline $\mathrm{In}_{0.86} \mathrm{Ga}_{0.14} \mathrm{As}_{0.3} \mathrm{P}_{0.7}$ & $45 \mathrm{~nm}$ \\
\hline $\begin{array}{l}\text { InGaAsP quaternary @ } \\
1100 n m\end{array}$ & $75 \mathrm{~nm}$ \\
\hline $\mathrm{N}-\operatorname{In} \mathrm{P}$ & $500 \mathrm{~nm}$ \\
\hline N+ InP Substrate & \\
\hline
\end{tabular}

\subsection{The top view of the device}

A schematic of the device is shown in Fig 2. It has a 5-um-wide ridge waveguide that consists of a 20um straight section and a 280um bending section. Chemical etching was used to create the ridge structure, but was stopped at about 0.1 um above the active layer. $\mathrm{A} \mathrm{SiO}_{2}$ insulation layer was deposited after the etching. Then, this layer was etched away on top of the ridge waveguide for $\mathrm{p}$-contact metallization. The fabrication was completed by $\mathrm{n}$-contact metallization and the devices were then cleaved apart.

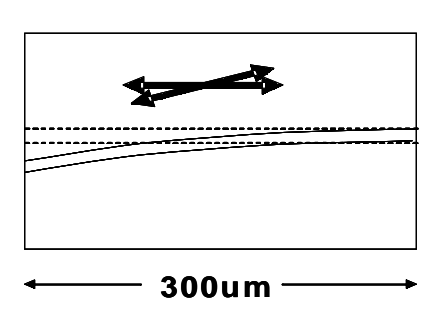

Fig 2. Schematic of the device. Two bi-directional arrows mean the directions of the propagation light.

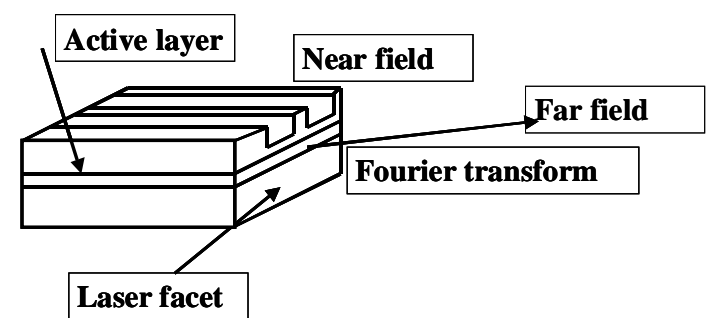

Fig 3. This graph describes the relationship between light near field pattern and the far field pattern.

\subsection{The experimental setup}

To understand the bi-directional lasing phenomenon in our bent waveguide laser diode, we do a series of experiments to investigate the actual physical origins. The spatial-intensity distribution of the emitted light near the laser facet is known as near field. The angular intensity distribution far from the laser facet is known as the far field (as shown is Fig 3 ). Thus, light far field pattern of the device is needed for light emission path study first. Second, the light 
spectrum helps us to compare the source of two emitted lights. Third, mirror feedback and grating feedback are chosen to see the lasing behavior affected by the external resonance cavity.

The experimental setup is shown in Fig 4(a) and Fig 4(b). In Fig 4(a), we measure the far field pattern of the device under different injection currents. The detector is used to record the every point of light intensity around the circle-like path. The emitting angle is also defined in the graph. Grating or mirror is used to feedback one direction of the light to see if the far field pattern will be changed. Meanwhile, collimators in the other side collect the light into optical fiber and the light is delivered to the optical spectrum analyzer (OSA), where we get the spectrum of lasing signal.

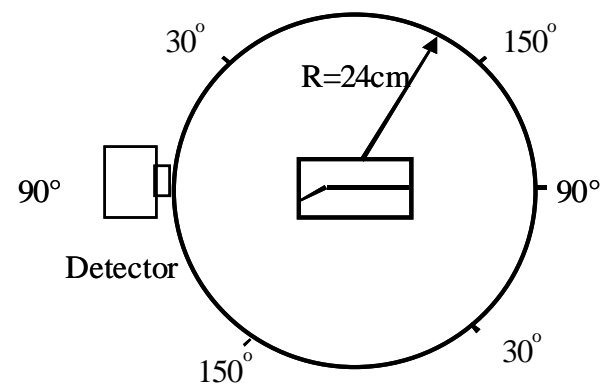

Fig 4(a) The detector sweeps and measures the far field pattern from small angle to big angle on both sides

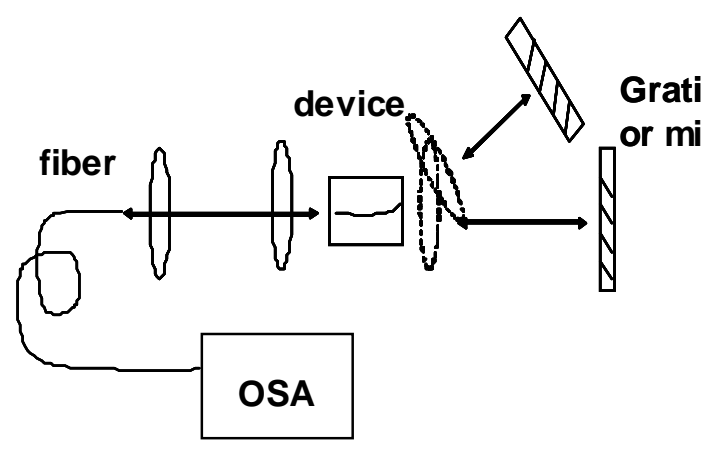

Fig 4(b). The experimental setup is used to measure the spectra under feedback mechanism. Far field pattern is directly measured by the detector.

\section{Results}

\subsection{Light Far field pattern}

Fig 5(a) shows the far field pattern of the bending side and Fig 5(b) shows the far field pattern of the straight side for different injection currents. When the current is not high enough ( it means the lasing condition is not reached ), the light disperses over a large range of angles and its intensity has smooth distribution ( Fig 5(c) and Fig 5(d) show more detail result ). If we increase the current to $350 \mathrm{~mA}$, the device starts lasing. Then there are two obvious light spots emitted from both sides of the device. Thus we can clearly see two main lobes of light on the far field pattern. Guiding mode effect is obvious when the threshold condition is reached. It means the waveguide has strong influence on the lasing light, leading to light path propagating along the waveguide direction, while spontaneous emission or amplified spontaneous emission field pattern is not shaped by the waveguide.

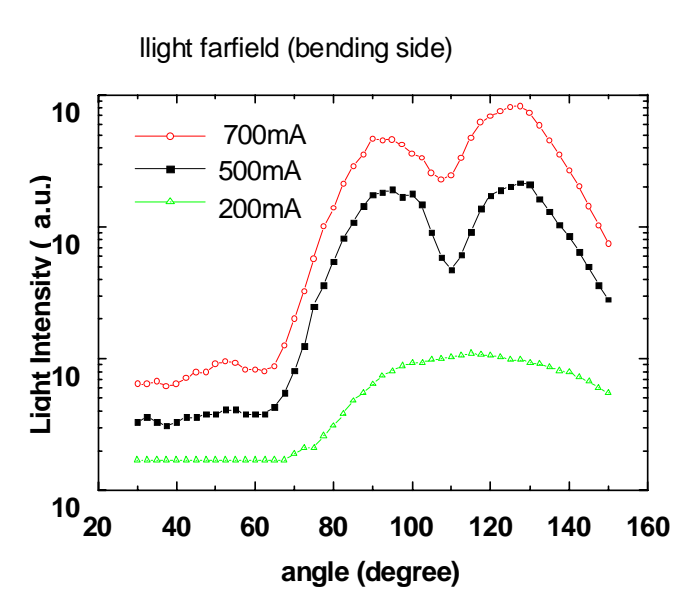

Fig 5 (a) Light far field pattern under different injection current measured in the bending side.

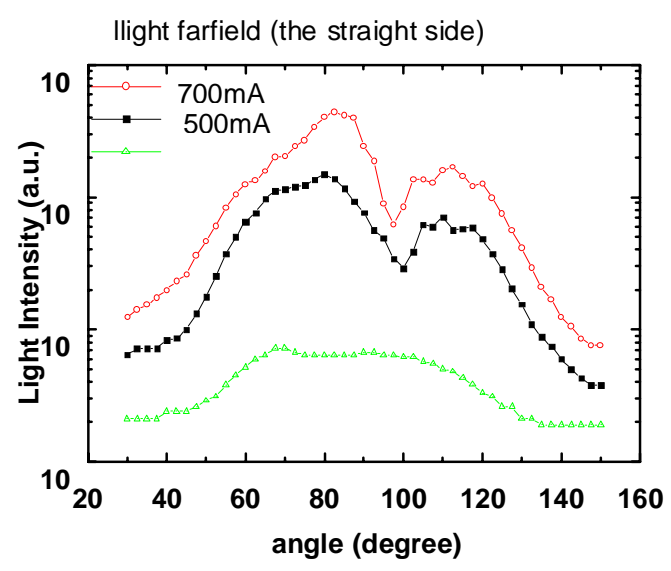

Fig 5 (b) Light far field pattern under different 


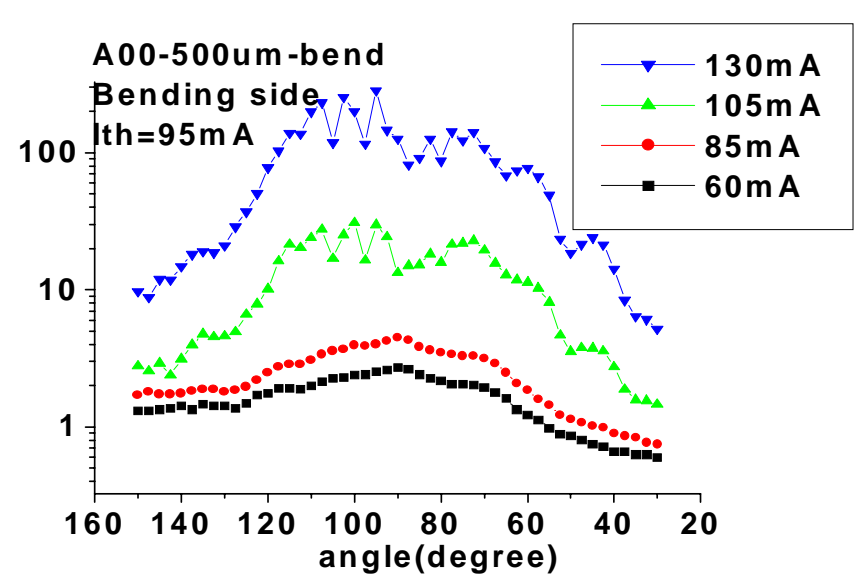

Fig 5 (c) Spontaneous or amplified spontaneous emission light far field pattern under different injection current measured in the

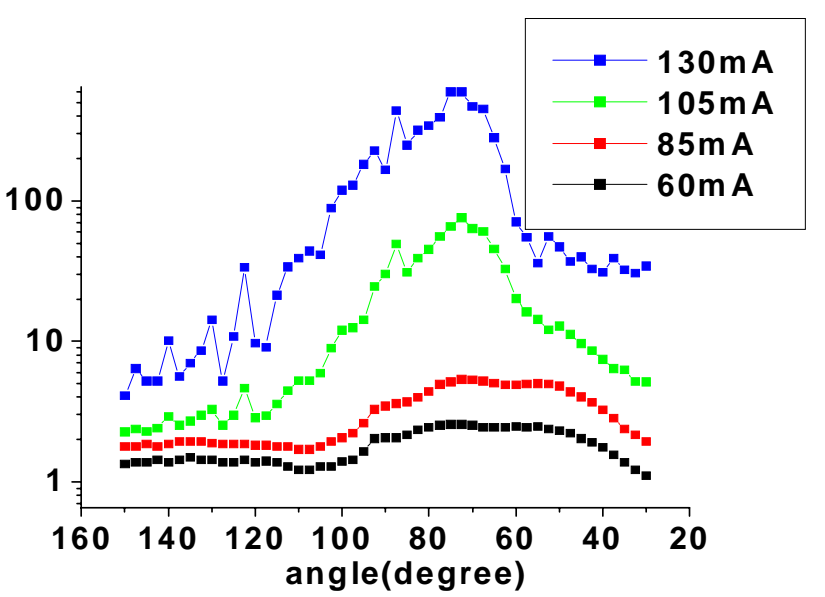

Fig 5 (d) Spontaneous or amplified spontaneous emission light far field pattern under different injection current measured in

\subsection{Spectra of the four ways of light}

Figs. 6 (a),(b),(c),(d) are the self-lasing spectra measured at different angles and different sides of the device. The comparison of Figs. 6(a) and (c) shows that the emission spectra from the straight side of the device are very similar, indicating that light at different directions come from the same source. The comparison of Figs. 6 (b) and (d) shows similar situation. The difference between them is that the intensity of the spectrum measured at the big angle is larger than that measured near rectangular angle. On the other hand, the two sides of the device have somewhat different spectra. It implies that the light from either the straight way or the bending way may be from the same source but couple to different paths, but the guiding behaviors from different sides are different. The spectra under different injection currents are shown in Fig. 6 (e) and Fig. 6 (f). For higher injection current, the spectra show that the lasing wavelength is blue shifted. 


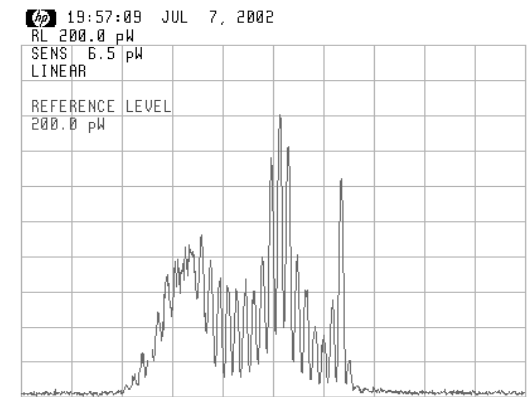

Fig 6 (a) The spectrum measured in the straight side at $115^{\circ}$ is scanned from $1300 \mathrm{~nm}$ to $1350 \mathrm{~nm}$.

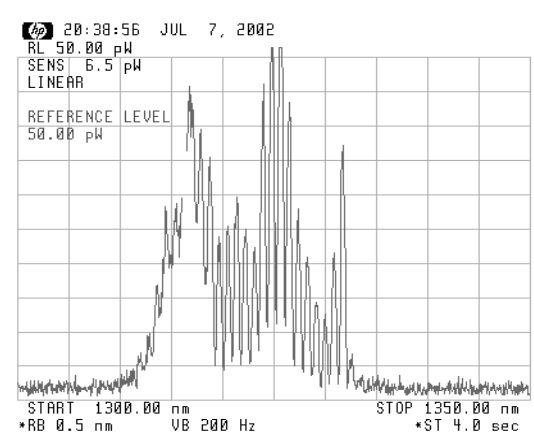

Fig 6 (c) The spectrum measured in the straight side at $85^{\circ}$ is scanned from $1300 \mathrm{~nm}$ to $1350 \mathrm{~nm}$.

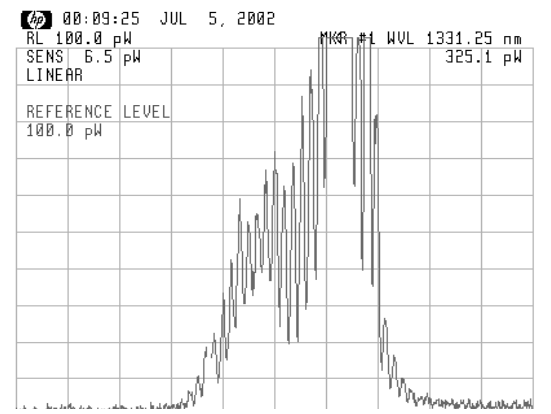

Fig 6 (b) The spectrum measured in the bending side at $125^{\circ}$ is scanned from $1300 \mathrm{~nm}$ to $1350 \mathrm{~nm}$.

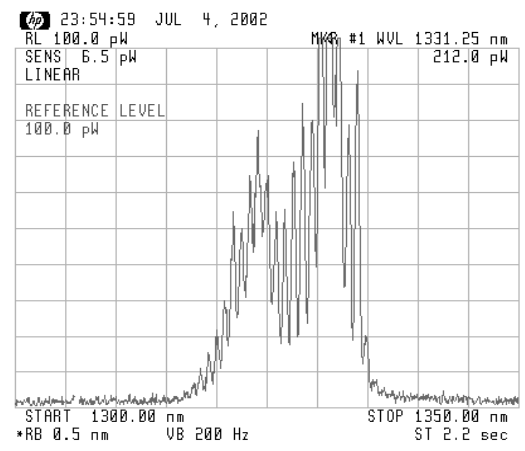

Fig 6 (d) The spectrum measured in the bending side at $95^{\circ}$ is scanned from $1300 \mathrm{~nm}$ to $1350 \mathrm{~nm}$.

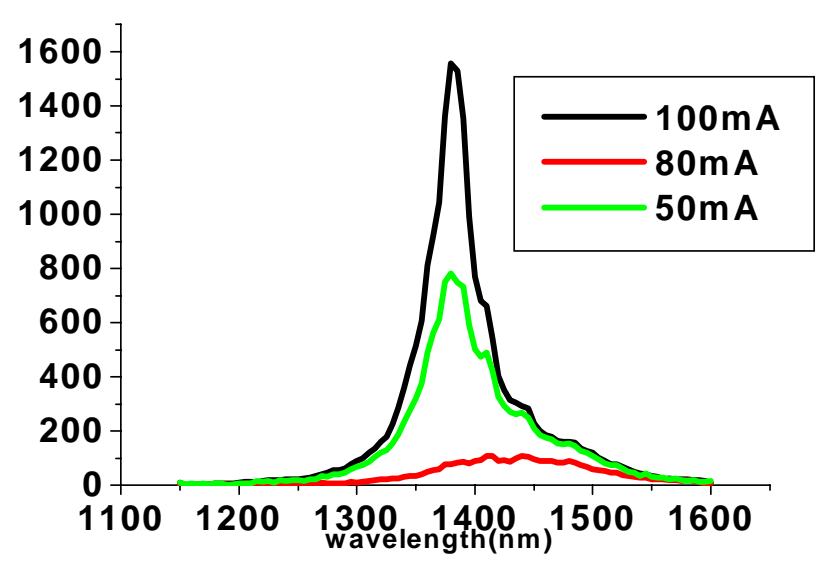

Fig 6 (e) The spectrum of the straight side under different injection current. The current from below to top is $80 \mathrm{~mA}, 100 \mathrm{~mA}, 120 \mathrm{~mA}$ 


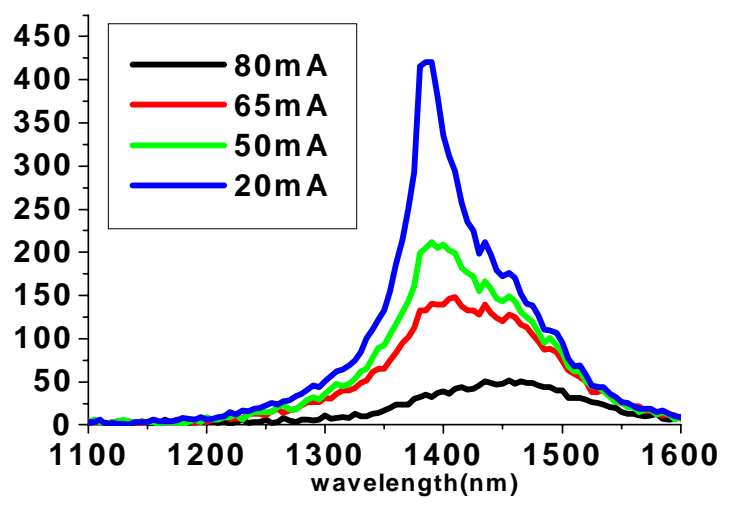

Fig 6 (f) The spectrum of the bending side under different injection current. The current from below to top is $80 \mathrm{~mA}, 90 \mathrm{~mA}, 100 \mathrm{~mA}, 120 \mathrm{~mA}$.

\subsection{Characteristics with mirror feedback}

It is interesting that from the light far field pattern, there are two paths for light to propagate. One path is considered as straight one and the other is tilted path. We use mirror feedback to give external influence to the inside of the device. Figs. 7 (a) and (b) show light far field patterns measured from the straight side when bending side is given feedback by the mirror at $125^{\circ}$ and $95^{\circ}$, respectively. From Fig 7 (a), the straight direction of the light is amplified. In contrast, from Fig. 7 (a), the light intensity at $120^{\circ}$ in the straight side seems to increase, but not obvious. On the other hand, the feedback by the mirror at $125^{\circ}$ does not have much influence, as shown by Fig. 7(b), although the light intensity at $120^{\circ}$ seems to increase slightly. Therefore, feedback with the mirror in the normal direction is stronger. It means the straight path is easy to have resonance. The different angles of feedback mechanism increase two different angles of light. This again proves that light goes in two kinds of paths, say, straight and bending way .

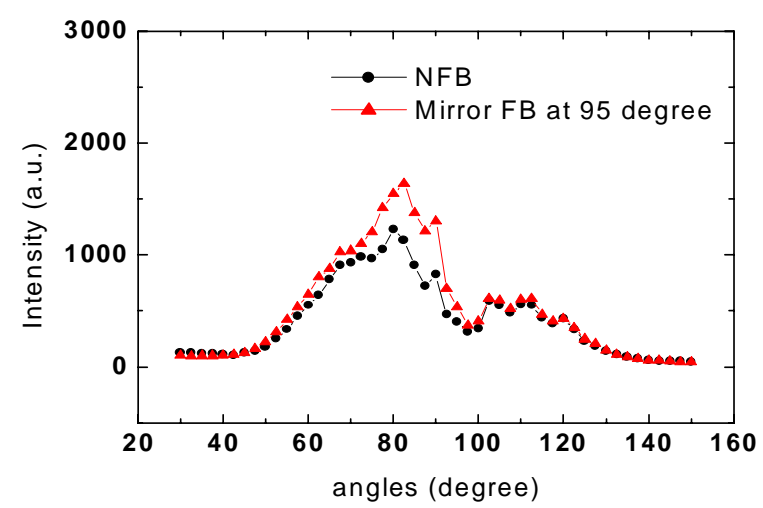

Fig 7 (a) Far field pattern using the mirror to feedback the light at $95^{\circ}$. NFB means that no feedback is used.

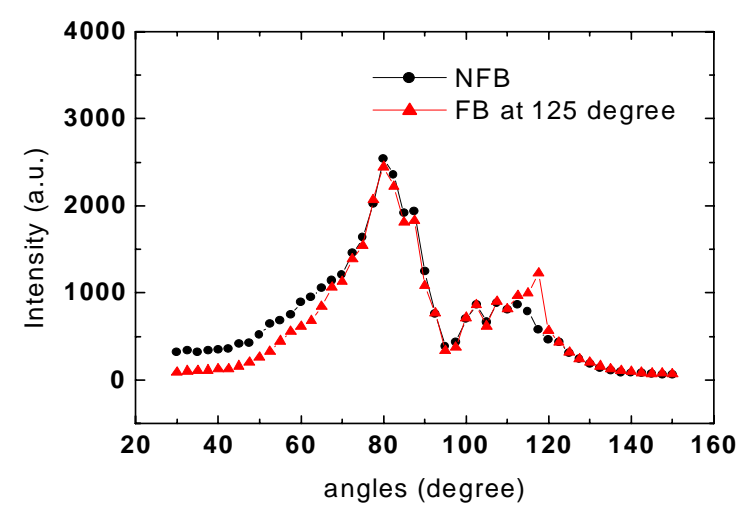

Fig 7 (b) This is the far field pattern using the mirror to feedback the light at $125^{\circ}$. NFB means that no feedback is used.

\subsection{Feedback wavelength selection with grating}

According to previous results, ${ }^{6}$ one can use grating to give external feedback at the desired wavelength in the bidirectional lasing system. The feedback wavelength will compete with the internal lasing wavelength and the cross switching effect occurs under different environmental condition. In experiment, we cannot give feedback at $125^{\circ}$ using grating because the loss in this path is more than the straight one. At $90^{\circ}$, we choose different wavelength to give feedback, the straight way is successfully tuned from $1305 \mathrm{~nm}$ to $1345 \mathrm{~nm}$. The self-lasing peak is at $1430 \mathrm{~nm}$. Here, the fact that resonance favors straight path is proved. The tuning range of the device is shown in Fig. 8 (a) and Fig. 8 (b). 


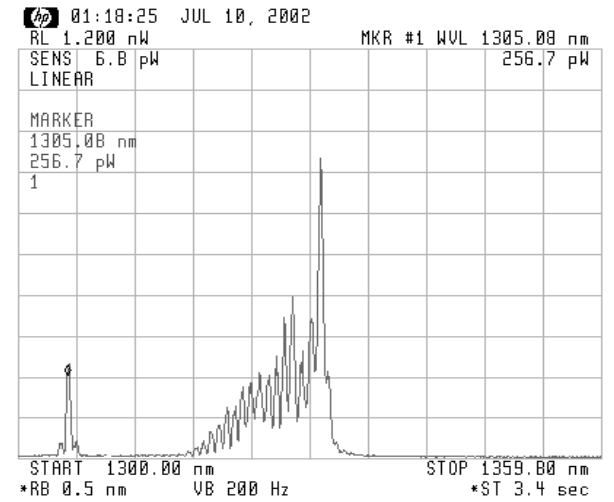

Fig 8 (a) The spectrum under grating feedback at $1305 \mathrm{~nm}$ is swept from $1300 \mathrm{~nm}$ to $1360 \mathrm{~nm}$.

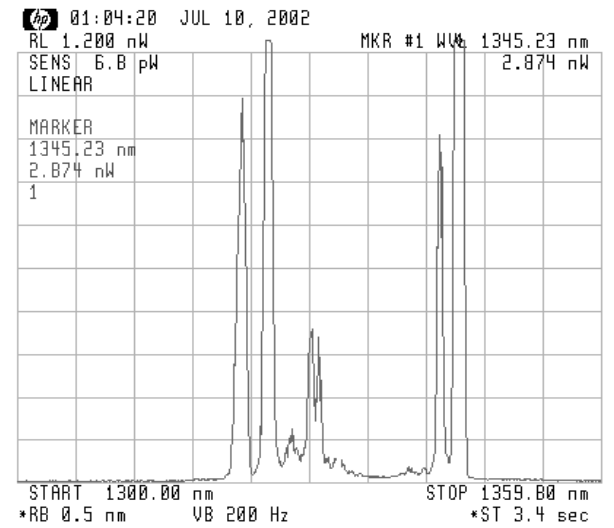

Fig 8 (b) The spectrum under grating feedback at $1345 \mathrm{~nm}$ is swept from $1300 \mathrm{~nm}$ to $1360 \mathrm{~nm}$.

\section{Discussion}

The bending waveguide structure is used to prevent the internal feedback. Thus resonance cavity will not exist in this device. However, in this experiment, we find the bi-directional guiding effect of the lasing mode in the bending ridge waveguide.

We look at the structure of the device, as shown in Fig. 9. Active layer consisting of the quantum wells is in the central region and there are two cladding layer beside them. Active region has higher refractive index compared to the cladding layer. Thus, light will be confined in the small region around the active layer. This is called optical confinement in the vertical direction. Above these layers, InP material is shaped as ridge layer to guide the light. The ridge waveguide has the thicker InP and leads to higher refractive index. It forms the optical confinement laterally. It should be noticed that there is a thin oxide layer between top metal and the ridge layer. ${ }^{7}{ }^{8} \mathrm{SiO}_{2}$ is used to block the injection current entering the whole active layer and lower the threshold current. Only the region under the waveguide has the current pumping and light propagating in active layer under this region obtains gain. Generally speaking, waveguide restricts the pumping region and decides the light propagation path.

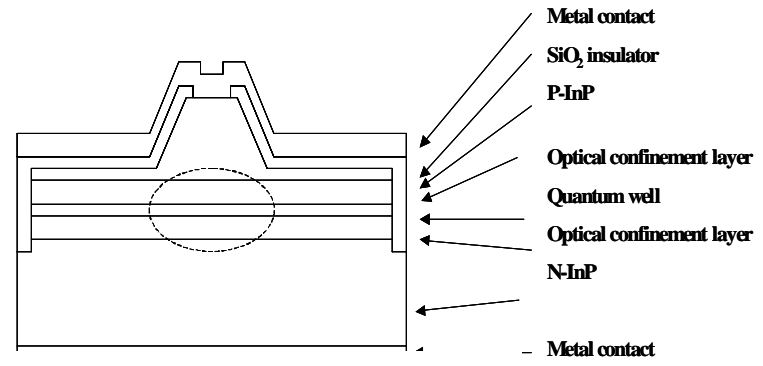

Fig 9. The structure of the laser diode

The experiment shows that it is easier to give feedback in the normal direction ( 90 degrees ) of the device, while the tilted way can not lase using grating feedback. The far field pattern with mirror feedback also tells us that the normal way is a better choice to reflect the emission light. Now we have to explain the lasing phenomenon on this bending structure waveguide. If we suppose the light have resonance along the ridge waveguide path, say bending path, it is contrary to the physical origin. Therefore, we claim the internal feedback is between two facets of the device. However, according to the above analysis, it seems impossible to have the light lasing along the straight path because this path is not directly pumped. Is that the real case? In fact, the injection current is not only restricted in the ridge waveguide. It will spread to some range around it. The large current spreading in the shallow-etched ridge waveguide 
causes the straight-propagation path that is close to the pumped bending waveguide to be partially pumped. Therefore, resonance along this path between the two cleaved facets could occur.

The shallow-etched depth leads to a small difference of the refractive indices between the ridge and its neighboring regions, so the guiding effect of the ridge waveguide is weak. ${ }^{3,5,9}$ The weak guiding effect then causes the light to possibly propagate along two paths. Due to the weak guiding effect, the light will not be well guided along the bending section when it propagates from the straight section to the bending section. Some portion of the light continues to propagate along the straight path, as indicated by the dashed lines in Fig 2, while the other portion is still guided along the bending ridge waveguide. Resonant mechanism occurs in the straight path and the light from straight path will couple to the bending waveguide. Therefore, we can detect the lasing light from bending direction. This results in two spots of maximum intensity in the light far field pattern. This also explains that the spectra of the lasing light from both angles in one side are almost the same, except the intensity of them is different. Similar spectra imply the same source of two directions of the light.

If the injection current does not reach threshold condition, the guided effect is not obvious. Also, the spreading current is not large enough to give sufficient gain for the neighborhood of the ridge waveguide. Neither light in the straight path be pumped to acquire enough gain. The behavior of the light far field pattern is like that of light emitting diode. Spontaneous emitted light is neither guided well in the bending waveguide nor going through the direct path between two facets. Light thus disperses at the big range of the angles. The far field pattern is very different before and after the device lases.

\section{Conclusion}

Bi-directional guiding effect of lasing mode is observed in a shallow-etched bending ridge waveguide. The lasing light propagates in two different paths, the straight way and the bending way. The far field pattern is quite different before and after the lasing condition is reached. This is because the bi-directional lasing guiding effect occurs. The experiment of giving feedback to the device with the mirror at different angles implies the way where light go through. Resonance occurs in the straight path, while the light in this path will couple to the bending waveguide and we can also detect the lasing light from bending direction.

\section{References}

1. N. A. Olsson, M. G. Oberg, L. D. Tzeng, and T. Cella, "Ultra-low reflectivity $1.5 \mathrm{~mm}$ semiconductor laser amplifier," Electron. Lett. 24, pp. 569-570, 1988.

2. A. T. Semenov, V. R. Shidlovski, and S. A. Safin, "Wide spectrum single quantum well superluminescent diodes at $0.8 \mathrm{~mm}$ with bent optical waveguide," Electron. Lett. 29, pp. 854-857, 1993.

3. Ching-Fuh Lin and Chaur-Shiuann Juang, Superluminescent diodes with bent waveguide," IEEE Photonics Technol. Lett. 8, pp. 206-208, 1996.

4. G. A. Alphonse, D. B. Gilbert, M. G. Harvey, and M. Ettenberg, "High-power superluminescent diodes,” IEEE J. Quantum Electron. 24, pp. 2454-2457, 1988.

5. Ching-Fuh Lin and Bor-Lin Lee "Bi-directional switching based on semiconductor laser with shallow-etched bending ridge waveguide" Appl. Phys. Lett. 71, 6 October 1997

6. Tiku-yu and Ching-Fuh Lin "Multiple cross switching effects in the bi-directional semiconductor laser" opt2001, Kaoshung, Taiwan R.O.C.

7. Semiconductor Lasers, Govind. Agrawal and Niloy L.Dutta,Van Nostrand Reinhold, New York 1993

8. A.E. Siegman, Lasers ( University Science Books, 1986 )

9. Ching-Fuh Lin, Bor-Lin Lee, and Po-Chien Lin, "Broadband superluminescent diodes fabricated on a substrate with asymmetric dual quantum wells,” IEEE Photonics Technol. Lett. 8,1456 (1996). 